\title{
Delivery Systems of Local Anesthetics in Dentistry: An Update
}

\author{
Neha Thilak ${ }^{1 *}$, Sundeep Hegde ${ }^{2}$, Sham S Bhat ${ }^{3}$, Vidya Bhat ${ }^{4}$, Subhathira \\ Rajasekaran ${ }^{\mathbf{1}}$ and Minu Mumtaz OV ${ }^{\mathbf{1}}$ \\ ${ }^{1}$ Post Graduate, Department of Pedodontics and Preventive Dentistry, Yenepoya Dental \\ College, Mangalore, Karnataka, India \\ ${ }^{2}$ Professor, Department of Pedodontics and Preventive Dentistry, Yenepoya Dental \\ College, Mangalore, Karnataka, India \\ ${ }^{3}$ Professor and HOD, Department of Pedodontics and Preventive Dentistry, Yenepoya \\ Dental College, Mangalore, Karnataka, India \\ ${ }^{4}$ Professor, Department of Prosthodontics, Yenepoya Dental College, Mangalore, \\ Karnataka, India
}

*Corresponding Author: Neha Thilak, Post Graduate, Department of Pedodontics and Preventive Dentistry, Yenepoya Dental College, Mangalore, Karnataka, India.
Received: March 17, 2020

Published: April 14, 2020

(C) All rights are reserved by Neha Thilak., et al.

\begin{abstract}
Effective delivery of local anesthesia is one of the most important aspects of dentistry. Obtaining adequate pain control will result in minimum discomfort of the patient during dental procedures, at the same time it helps the dentist to render the treatment effectively. Even though the modern dental syringes which is the most common method of local anesthesia administration were introduced many years ago, different modifications in delivering local anesthetics have evolved over time. These innovations have lead to decreased pain during administration of local anesthesia and also reduced side effects. This article discusses about different advancements in the delivery systems of local anesthesia.
\end{abstract}

Keywords: TENS; CCLAD; Intra-Osseous Anesthesia; Local Anesthesia; STA; Wand

\section{Abbreviations}

TENS: Transcutaneous Electric Nerve Stimulation; CCLAD: Computer Controlled Local Anesthetic Delivery System; STA: Single Tooth Anesthesia; TAC: Tetracycline, Adrenaline, Cocaine; LET: Lidocaine, Epinephrine, Tetracaine; CHADD: Controlled Heat Aided Drug Delivery Patch; EMLA: Eutectic Mixture of Local Anesthetics

\section{Introduction}

Pain is always a matter of concern in delivering effective dental treatment especially in children and thus pain control forms the foundation of successful management of patients in dentistry [1]. Pain in the dental operatory can influence the attitude towards dentistry negatively, which in turn leads to dental anxiety and fear in the future dental visits of a child and hence, it is important for pediatric dentists to minimize the same. Majority of the procedures in dentistry causes some sort of discomfort or pain which can be alleviated by the administration of local anesthesia, which itself provokes pain [2].
Success of modern dental practice is determined by efficacy of local anesthetic drugs and delivery of local anaesthesia. Fear of injection is the main reason for most of the people to refuse dental treatments. Newer technologies have lead to decreased pain during administration of local anesthesia and also reduced side effects. This article discusses about different advancements in the delivery systems of local anesthesia.

\section{Topical anaesthetics}

Topical anaesthesia is that form of anaesthesia obtained by the direct application of the drug to abraded skin or to the mucous membrane [3]. It is used to anesthetize small wounds and are available in different forms such as gels, lotions, patches, cream, solutions, aerosol forms. This type of anesthesia is mainly useful in children because of the ease of application and less pain due to the absence of needle [4]. 
According to the vehicle used, topical anaesthetics are classified as non-aqueous or water insoluble and water soluble.

Non aqueous topical anesthetics

These anaesthetics are insoluble in water but they are soluble in vehicles such as alcohol, polyethylene glycol, propylene glycol or carboxymethylcellulose. They include Benzocaine and Lidocaine base:

Benzocaine: It is an ester of amino-benzoic acid that lacks a cationic amino terminus which makes benzocaine poorly soluble in water and a poor candidate for parenteral use. It is available in the form of solution as $6-20 \%$ and also as spray, gel, gel-patch, ointment. They are also available in different flavours. Brand names available: Orajel, Hurricaine.

Combinations of benzocaine includes Orabase (combination of benzocaine, gelatin, pectin and sodium carboxymethyl cellulose), Cetacine (14\% benzocaine, $2 \%$ butamen, $2 \%$ tetracaine $\mathrm{Hcl}$ ) [5].

Hurripak is a recent advancement which contains 20\% benzocaine, an anaesthetic liquid which is one of the recent development topical anaesthetics for periodontal procedures. It is available as a needle free kit. It is inserted deep within the gingival sulcus. The onset and the duration of action are 30 seconds and 15 minutes respectively [5]. Multiple administrations are recommended because of its inefficacy in providing adequate anesthesia in single administration [4].

Lidocaine: Lidocaine base is used in 5\% concentration and used in case of minor burns, abrasions of skin, applied to mucous membrane of oropharynx, that is accessible and provide temporary relief of pain associated with it. It contains lidocaine and polyethylene. It is available as flavoured gels and ointments. Brand name includes Lidocream [3].

\section{Water soluble topical anaesthetics}

These anaesthetics are soluble in water. It will rapidly get absorbed into the blood stream and thus toxic reactions can be expected like allergic reaction, methemoglobinemia etc. So, it is not recommended to be sprayed on mucous membrane. This group includes benzyl alcohol, tetracaine hydrochloride, lidocaine hydrochloride, combinations of tetracaine hydrochloride which includes Tetracaine, adrenaline (epinephrine), and cocaine (TAC), Lidocaine, epinephrine, and tetracaine (LET) [4].

S-caine patch ${ }^{\mathrm{TM}}$ and local anesthetic peel the patch

It is a controlled heat aided drug delivery patch (CHADD) with a heat generating medium, a drug reservoir for lidocaine and tetracaine and a medical tape cover. The application of warm technology helps in accelerating transcutaneous delivery and analgesic effect of anaesthetics [6].

Lidocaine hydrochloride

It is an amide based topical anesthetic which is available as Lidoderm patch comprised of an adhesive material containing $5 \%$ lignocaine. Dentipatch and Topicaine (4\% lignocaine in a hydroethanolic gel microemulsion) are also available [4].

Eutectic mixture of local anaesthetics (EMLA) is an oil-inwater emulsion mixture

It is composed of lignocaine $2.5 \%$ and prilocaine $2.5 \%$ in an emulsion in which oil phase is a eutectic mixture of lignocaine and prilocaine in a ratio of $1: 1$ by weight [7]. It has got low viscosity hence there is difficulty in handling. So, it is applied under occlusive dressings like Tegaderm ${ }^{\mathrm{TM}}$, Saran-wrap ${ }^{\mathrm{TM}}$, or Band-aid ${ }^{\mathrm{TM}}$, which aids diffusion into the skin. Maximal depth of anesthesia is $3 \mathrm{~mm}$ after a 60-minutes application, and $5 \mathrm{~mm}$ after a under occlusive dressing and action persist for 1 - 2 hours [4].

\section{Betacaine}

It contains lignocaine, prilocaine and phenylephrine. Concentrations of lignocaine and prilocaine is found to be 4 times that of EMLA and so, it should not be applied to an area larger than $300 \mathrm{~cm}^{2}$ and is not advocated for use in children [8].

Oraqix

It contains $2.5 \%$ of lidocaine and $2.5 \%$ of prilocaine. It is deposited into periodontal pockets during root planing and scaling. Reports of its efficacy of pain relief during orthodontic band placement are well documented [9].

Recent advances in topical anaesthesia

- Precooling (Cryo Anaesthesia): It is the application of cold to a localised part of body in order to block the local nerve conduction of painful impulses [10]. It stimulates myelinated A fibers, activating inhibitory pain pathways which in turn raises pain threshold. A study reported that cooling will slow down or eliminate transmission of pain signals [11]. It is available in ice (crushed ice or cubed ice), refrigerant spray forms. The trade names of refrigerant spray are Gebauer's pain ease, Pharma ethyl. It is short acting and application time is 2 - 5 minutes [12].

- Iontophoresis: It enhances the transport of topically applied drugs using a mild electric current so that permeability of drugs that are charged can be increased through the skin [13]. 
Transcutaneous electric nerve stimulation (TENS)/electronic dental anaesthesia

It is a widely used non pharmacological technique in managing acute and chronic pain. Transcutaneous electrical nerve stimulation (TENS) uses electric current produced by a device for the stimulation of nerves mainly for therapeutic purposes. The equipment does not contain any syringes because of which it imparts a positive behaviour in children and reduces their fear. Hence, this technique can be used successfully in pediatric patients. It can be also be equally useful in adult patients to produce analgesia during different conditions like rubber dam placement, cavity preparation, pulp capping, endodontic procedures, prosthetic tooth preparations, oral prophylaxis, extractions, and also to reduce the discomfort during injection of local anaesthesia [4].

It consists of a TENS unit which is an electric pulse generator with two variations- a model used by the dentist in the clinic which is connected to the buildings electrical outlet to generate power. Next variation is patient model, which is small and portable type with battery as a power source. In addition to the TENS unit, it consists of lead wires and also electrodes that can be placed either extraorally or intraorally [4].

A study compared efficacy of TENS with $2 \%$ lignocaine in reducing pain during extraction, cavity preparation, pulpotomy and pulpectomy of deciduous teeth and concluded that TENS can be a useful adjunct in pediatric patients in achieving anaesthesia [14].

Computer controlled local anaesthetic delivery system (CCLAD)

CCLAD renders painless anaesthesia by administering small amount of anaesthetic solution at slow speed. It can be used successfully in pediatric patients. It can be equally indicated in adult patients to produce anaesthetic effects during pulp capping, endodontic procedures, extractions. It is indicated for delivery of painless palatine injections. Trade names include Compudent, Comfort control syringe ${ }^{\circledR}$, Single tooth anaesthesia ${ }^{\mathrm{TM}}$, Quick sleeper $^{\circledR}$, Sleeper one ${ }^{\circledR}[4]$.

Compudent/wand computer controlled local anaesthetic system $^{\circledR}$

It was originally known as Wand. Later, subsequent versions were introduced in the market as Wand Plus and then CompuDent, the current designation. It consists of a base unit, hand piece and foot pedal. The solution is forced through the micropore tubing of the handpiece controlled by computer control unit. Upon activation of foot pedal, rate of injection can be controlled. Three modes of flow rate are available namely slow, fast and turbo speed [15].

\section{Comfort control syringe ${ }^{\circledR}$}

This modification improves on the CCLAD concept. It is an electronic, preprogrammed delivery system that provides the operator with the control needed to make the patient's local anesthetic injection experience as pleasant as possible. It has a two-stage delivery system; the injection begins at an extremely slow rate to prevent the pain associated with quick delivery. After 10 seconds, speed increases to the preprogrammed injection rate for the technique selected [16]. There are five preprogrammed injection rates for specific injections:

- Infiltration - $0.007 \mathrm{ml} / \mathrm{sec}$

- Regional block - $0.02 \mathrm{ml} / \mathrm{sec}$

- Palatal - $0.008 \mathrm{ml} / \mathrm{sec}$

- Intraligamentary- $0.007 \mathrm{ml} / \mathrm{sec}$

- Intraosseous injections - $0.02 \mathrm{ml} / \mathrm{sec}$ [17].

Single tooth anaesthesia system (STA) ${ }^{\mathrm{TM}}$

STA uses vibration technology to decrease the pain in addition to the computer controlled delivery. It has a pressure sensing technology. This assesses the exit pressure of solution. STA includes foot pedal, cartridge holder and tube. Injection rate can be selected from the unit [15].

Quick sleeper ${ }^{\circledR}$

It is an alternative for Wand. It includes hand piece with a grip, foot pedal, control unit, and a permanent analysis of resistance system. Quantities of anaesthetic solution injected can be directly viewed all over the hand piece. A new model Quick Sleeper S4 was introduced which is $40 \%$ lighter and 19\% reduced in diameter compared to other models [18].

\section{Sleeper one ${ }^{\circledR}$}

The design is similar to Quicksleeper. It is composed of a pen grip hand piece, a control unit, a foot pedal and permanent analysis of resistance system. It has got a recapping system on the handpiece holder so that accidental injuries can be prevented. The design of needles allows easy penetration into every type of tissue, without effort, without twisting and without pain [4].

\section{Vibrotactile devices}

Vibrations or pressure act as non-noxious stimuli, which modify or interfere with pain signals by closing the neural gate of cerebral cortex. As cerebral cortex gets focused on vibration, there is less perception of pain. Studies suggested that pain reduction due to 
non-noxious touch or vibration results from tactile induced pain inhibition within the cerebral cortex itself and that the inhibition occurs without any contribution at spinal level, including descending inhibitory actions on spinal neurons [19]. Vibrotactile devices includes vibraject, dentalvibe, accupal.

\section{Vibraject $^{\circledR}$}

Vibraject is a battery operated device which has an attachment that snaps on to the standard dental syringe. It produces vibrations at high frequency on to the needle which inhibits pain sensation at the time of injecting anesthetic [17].

\section{Dentalvibe $^{\circledR}$}

DentalVibe is a handheld cordless injection system, with a U-shaped tip that vibrates which is attached to a microprocessorcontrolled Vibra-Pulse motor. It has got an attachment which will help to retract the lip or cheek [17].

\section{Accupal $^{\circledR}$}

The Accupal is a cordless battery operated device used for inferior alveolar blocks and palatal injections. It uses vibration as well as pressure so that the alveolar or palatal mucosa gets preconditioned. It also provides pressure and vibrates the injection site, $360^{\circ}$ proximal to the needle penetration that shuts the pain gate mechanism which blocks the pain sensation [17].

\section{Jet injection}

In jet-injection technology, pressure is created with the help of mechanical energy in order to push the solution out through the orifice. It is fast and easy to use, with little or no pain, less tissue damage, faster drug absorption and less chances of infection at the site. It is mainly of use in children because of less bone density [17].

\section{Syrijet $^{\circledR}$}

The SyrijetMark II jet injector uses the standard 1.8-ml cartridges of local anesthetic solution which permits the administration of a variable volume of solution from 0 to $0.2 \mathrm{ml}$. Nozzle pressure is 2000 pounds per square inch (psi) hence there is a good penetration capability, with quantities up to $0.2 \mathrm{ml}$ per injection. It is mainly used in children [17].

\section{Med-Jet $^{\circledR}$}

Med-Jet is a needle-free injection system. It uses compressed air for injection delivery. Solution is forced through the mucosa by placing the head of the device against it and by releasing the trigger. Intradermal, subcutaneous or intramuscular injections can be given with a volume of 0.01 to $1 \mathrm{cc}$. at 2000 psi. It is painless. It is used for nasopalatine and greater palatine injections but inadequate for pulpal block. It is very successful in children. A few drawbacks include hematoma at the site of anesthesia and high cost [17].

\section{Safety dental syringe}

Safety dental syringe minimizes the risk of accidental needle-stick injury occurring to a dental health provider with a contaminated needle after the administration of local anesthesia. These syringes possess a sheath that 'locks' over the needle when it is removed from the patient's tissues thereby preventing accidental needle stick injury. It includes ultra safe syringe, ultra safety plus XL syringe, hyposafety syringe, safety wand, Rev Vac safety syringe [20].

\section{Intra-osseous anesthesia}

The intraosseous injection allows placement of a local anesthetic solution directly into the cancellous bone adjacent to the tooth to be anesthetized. Infiltration injections with lidocaine solutions are not effective for anesthesia of the mandibular molar teeth due to the thickness of the cortical plate, hence dentists do not attempt infiltration anesthesia in the posterior mandible. The intraosseous injection overcomes this problem by allowing direct access to the cancellous bone. Several systems have been developed to achieve intra-osseous anesthesia. All these systems inject local anesthetic solution into the cancellous bone adjacent to the apex of the tooth. These systems are: Stabiden $t^{\circledR}, \mathrm{X}$ tip $^{\circledR}$ and Intraflow ${ }^{\circledR}[21]$. Stabident delivery system has a disadvantage that it can be used only in areas that are visible and readily accessible. Because of this reason, $\mathrm{x}$ tip uses pilot drill, hollow tube through which a 27 -gauge needle can pass. The initial drill stays in place, which allows the anesthetic to be placed without hunting for the hole. IntraFlow anesthesia system uses a single-step method with entry into the penetration area, injection, and withdrawal in one step [22].

\section{Conclusion}

Dentistry is fortunate in that it possesses an abundance of excellent agents for the relief of preoperative and postoperative pain associated with the delivery of dental care. Local anesthetics have made a great advancement in dentistry and have changed patients' perspectives of dental procedures to a great extent. A clinician's ability to administer an effective, safe and atraumatic local anesthesia injection to a child (or adult) is a major factor in creating a patient with a life long acceptance of dental treatment. Rather than avoiding local administration for fear of traumatizing the pediatric patient, the clinician should strive to learn and use the latest modalities of local pain control to create a pleasant and comfortable dental experience for the patient. 


\section{Bibliography}

1. Lee Hyo-Seol. "Recent advances in topical anesthesia". Journal of Dental Anesthesia and Pain Medicine 16.4 (2016): 237-244.

2. Malamed Stanley F. "Handbook of local anesthesia-e-book". Elsevier Health Sciences (2014).

3. Monheim Leonard M and Charles Richard Bennett. "Monheim's local anesthesia and pain control in dental practice". CV Mosby (1984).

4. Menon HM., et al. "Recent advances in local anesthesia". International Journal of Advanced Research 7.10 (): 734-760.

5. Boyce Ricardo A., et al. "Updates of topical and local anesthesia agents". Dental Clinics 60.2 (2016): 445-471.

6. Tadicherla Sujatha and Brian Berman. "Percutaneous dermal drug delivery for local pain control". Therapeutics and Clinical Risk Management 2.1 (2006): 99.

7. Daneshkazemi A., et al. "Efficacy of eutectic mixture of local anaesthesia as a topical anaesthetic agent used for dental procedure: A brief review". Anesthesia Essays and Researches 10.3 (2016): 383-387.

8. Friedman Paul M., et al. "Topical anesthetics update: EMLA and beyond". Dermatologic Surgery 27.12 (2001): 1019-1026.

9. Kwong Thomas S., et al. "The effectiveness of Oraqix versus TAC (a) for placement of orthodontic temporary anchorage devices". The Angle Orthodontist 81.5 (2011): 754-759.

10. Lathwal Garima., et al. "Efficacy of different precooling agents and topical anesthetics on the pain perception during intraoral injection: a comparative clinical study". International Journal of Clinical Pediatric Dentistry 8.2 (2015): 119.

11. Mohiuddin Iqra., et al. "Topical application of local anaesthetic gel vs ice in pediatric patients for infiltration anaesthesia”. Journal of Evolution of Medical and Dental Sciences 4.74 (2015): 12934-12940.

12. Aminabadi Naser Asl and RM Farahani. "The effect of pre-cooling the injection site on pediatric pain perception during the administration of local anesthesia". The Journal of Contemporary Dental Practice 10.3 (2009): 43-50.

13. Priyatham Sunny and Sivakumar Nuvvula. "Intraoral Topical Anaesthesia In Pediatric Dentistry". International Journal of Pharma and Bio Sciences 7.4 (2016): 346-353.
14. Dhindsa Abhishek., et al. "Comparative evaluation of the effectiveness of electronic dental anesthesia with $2 \%$ lignocaine in various minor pediatric dental procedures: A clinical study". Contemporary Clinical Dentistry 2.1 (2011): 27.

15. Clark Taylor M and John A Yagiela. "Advanced techniques and armamentarium for dental local anesthesia". Dental Clinics 54.4 (2010): 757-768.

16. Christopher A and Shankar Rohini. "Recent advances in local anesthesia - A review". International Journal of Recent Scientific Research 7(2016) :13576-13581.

17. Singh Nitin., et al. "Painless anesthesia: A new approach". Journal of Dentofacial Sciences 2 (2013): 49-55.

18. Tom Kaitlyn and Johan Aps. "Intraosseous Anesthesia as a primary technique for local anesthesia in dentistry". Clinical Research in Infectious Diseases 2.1 (2015): 1012.

19. Inui Koji., et al. "Temporal analysis of cortical mechanisms for pain relief by tactile stimuli in humans". Cerebral Cortex 16.3 (2006): 355-365.

20. Saxena Payal., et al. "Advances in dental local anesthesia techniques and devices: An update". National Journal of Maxillofacial Surgery 4.1 (2013): 19.

21. Reader AL and John Nusstein. "Local anesthesia for endodontic pain". Endodontic Topics 3.1 (2002): 14-30.

22. Kumar S. "Newer delivery systems for local anesthesia in dentistry". Journal of Pharmaceutical Sciences and Research 7 (2015): 252-255.

\section{Assets from publication with us}

- Prompt Acknowledgement after receiving the article

- Thorough Double blinded peer review

- Rapid Publication

- Issue of Publication Certificate

- High visibility of your Published work

Website: www.actascientific.com/

Submit Article: www.actascientific.com/submission.php Email us: editor@actascientific.com

Contact us: +919182824667 